Orte, C., Quesada, V., Valero, M. y Pozo, R. (2022). La Entrevista Motivacional en Programas Socioeducativōs Familiares con Adolescentes: una revisión sistemática. Revista de Investigación Educativa,40(1),127-145.

DOI: http://dx.doi.org/10.6018/rie.435031

\title{
La Entrevista Motivacional en Programas Socioeducativos Familiares con Adolescentes: una revisión sistemática
}

\section{Motivational Interviewing in socio-educational family programs with adolescents:A systematic review}

\author{
Carmen Orte,Victoria Quesada', María Valero, Rosario Pozo \\ Departamento de Pedagogía y Didácticas Específicas. Universitat de les Illes Balears
}

\begin{abstract}
Resumen
Los programas socioeducativos familiares son estrategias recomendadas para la prevención y promoción de comportamientos saludables en adolescentes. La entrevista motivacional (EM) pretende fortalecer la motivación y el compromiso para el cambio. La finalidad de esta revisión es examinar los programas con un componente familiar y socioeducativo para adolescentes que incorporan la EM con el objetivo de analizar sus características, cómo se integra la EM, la formación de los profesionales que la implementan y los resultados obtenidos en efectividad y retención. Para ello, siguiendo el protocolo PRISMA, se realiza una búsqueda bibliográfica en las principales bases de datos científicas de documentos publicados entre 1999 y 2019. Un total de 20 estudios cumplieron con los criterios de inclusión y son analizados en este estudio. Los resultados muestran que la EM se utiliza, con mayor frecuencia, de forma integrada en los programas y en formato presencial. Se aprecia variedad en el perfil de los profesionales que la aplican y una intensa formación. No se observan mejoras en la retención de los participantes, aunque sí obtienen resultados positivos en comportamientos saludables. Se requiere mayor reporte de investigaciones para poder localizar los efectos específicos de la introducción de la EM en este tipo de programas.

Palabras clave: Entrevista motivacional; programas familiares; educación; adolescentes; revisión sistemática.
\end{abstract}

1 Correpondencia: Victoria Quesada, victoria.quesada@uca.es, Universitat de les Illes Balears (España). 


\begin{abstract}
Socio-educational family programs are recommended strategies for prevention and the promotion of healthy habits in adolescents. Motivational interviewing (MI) aims to enhance motivation and commitment to change. Thus, the objective of this review is to examine socioeducational and family-based programs for adolescents that incorporate MI, in order to analyze their main features, MI integration, training of MI professionals, and results in effectivity and retention. To do so, following the PRISMA guidelines, a systematic review was made by searching the main scientific databases and taking studies published between 1999 and 2019. A total of 20 studies were selected and analyzed in this paper. Results show that MI is mostly face-to-face and embedded in the programs. There is a variety in the profiles of MI professionals and a thorough training. Although there is no improvement in participants' retention levels, positive results in their healthy habits are obtained. More research is needed to determine the specific effects of MI application in socio-educational programs.

Keywords: Motivational interviewing; family programs; education; adolescents; literature review.
\end{abstract}

\title{
Introducción
}

La Entrevista Motivacional (EM) es una guía colaborativa y centrada en la persona que busca promover y fortalecer la motivación para el cambio (Miller y Rollnick, 2015). A pesar de tener su origen en la práctica clínica y terapéutica, actualmente se emplea en diversas áreas, destacando su aplicación en contextos relacionados con la prevención de conductas problemáticas y de riesgo, la promoción de la salud y la educación en estilos de vida saludables. La EM es una estrategia prometedora para movilizar la motivación para el cambio de comportamientos de las personas en programas educativos y socioeducativos de prevención y promoción de la salud (Ballester et al., 2021; Dishion et al., 2002; Naar et al., 2019), así como en contextos educativos formales (Wells et al., 2014; Wells y Jones, 2018). Destaca especialmente su potencial para ayudar a establecer objetivos y resolver ambivalencias abordando las resistencias individuales, es decir, aquellos pensamientos, creencias o hábitos que hacen que se mantenga en la situación en la que se encuentra.

La prevención es una de las mejores opciones para evitar la aparición de posibles problemas, siendo la más económica a largo plazo. Existen tres niveles de prevención: 1) Universal o primaria, dirigida a toda la población, 2) Selectiva o secundaria, orientada a población que se encuentra en riesgo e 3) Indicada o terciaria, orientada a personas que ya presentan una problemática concreta. Aunque toda la población se puede beneficiar de estrategias preventivas, se obtienen mayores beneficios en grupos vulnerables o en riesgo (Norberg et al., 2013).

La familia es el referente prioritario del enfoque preventivo. Los programas socioeducativos familiares son estrategias recomendadas a nivel internacional para la prevención y/o reducción de comportamientos no saludables en adolescentes, como, por ejemplo, los consumos (alcohol, drogas, pantallas, etc.) (UNODC, 2015, 2018) o la obesidad (Smith et al., 2015), entre otros. Éstos buscan lograr cambios de conductas, 
desarrollando hábitos saludables. Lograr estos cambios es fundamental para conseguir una adecuada prevención de riesgos en etapas como la adolescencia.

Debido a la alta demanda de dedicación, los programas familiares a menudo tienen problemas para conseguir buenos niveles de adherencia (Negreiros et al., 2019; Orte et al., 2017), entendida como la asistencia continuada (retención), participación activa y empleo de las herramientas proporcionadas durante los programas por parte de los participantes. Así, conseguir vincular a los miembros de la familia con el proceso de cambio y que lo asuman como propio es un reto y un elemento en el que la EM puede resultar clave.

Diversos estudios han explorado si la aplicación de la EM y sus fundamentos pueden facilitar la implicación de las familias en los programas preventivos y socioeducativos mejorando los niveles de adherencia y de eficacia de las intervenciones (Belmontes, 2018; Gearing et al., 2014). En este sentido, aunque en otros contextos, Shaw y Taraban (2016) señalan que la EM es un componente importante que garantiza el éxito en retención, esto es, conseguir que los participantes terminen el programa, especialmente en periodos de transición como la adolescencia, dónde los padres pueden percibir más dificultades para gestionar el comportamiento de sus hijos.

Las revisiones y meta-análisis más recientes sobre la EM (Barnett et al., 2012; Cushing et al., 2014; Galvez et al., 2019; Gayes y Steele, 2014; Jensen et al., 2011; Lundhal et al., 2010) destacan sus efectos positivos en comparación con otras intervenciones y enfatizan la necesidad de analizar los aspectos relativos a modalidad de administración (presencial o telefónica), número de sesiones, formato del feedback, formación de los profesionales, etc. Sin embargo, ninguna de ellas estudia la aplicación de la EM en los programas familiares con adolescentes y desde una perspectiva socioeducativa. Es por ello que la finalidad del presente estudio es realizar una revisión sistemática de la literatura sobre la EM en programas familiares para adolescentes con un componente socioeducativo. Con ello, se pretende contribuir a una educación basada en la evidencia.

\section{Objetivos}

El objetivo principal es conocer qué intervenciones y programas socio-educativos con un componente familiar que utilizan la EM existen para población adolescente, analizar la forma en que se desarrollan y cómo se integra la EM en cada uno de ellos. Además de examinar las características de dichos programas, se persigue analizar el perfil de los profesionales que los implementan, la formación en EM que han recibido y los resultados obtenidos en eficacia y participación.

\section{Método}

Se realizó una revisión sistemática. Tres investigadoras revisaron en paralelo las principales bases de datos en educación entre febrero y julio de 2019: Web of Science (WOS), Scopus, ERIC, EbsCo y DIALNET. Además, dada su relevancia en difusión y como fuentes de información complementaria, se realizó una búsqueda en la red social de investigadores Researchgate y en el buscador académico Google Scholar. Asimismo, se revisaron los artículos que aparecían en las referencias bibliográficas de las revisiones sistemáticas y meta-análisis localizados. 
Para la revisión se siguió el protocolo PRISMA (Transparent Reporting of Systematic Reviews and Meta-Analyses) (Moher et al., 2009). La búsqueda se realizó en inglés, y las palabras clave utilizadas fueron: family program, family-based, family-centered, prevention, intervention, motivational interviewing y adolescents. La ecuación de búsqueda [("Family program" OR "family-based" OR "family-centered") AND (prevent ${ }^{*}$ OR intervent $\left.{ }^{*}\right)$ AND ("motivational interview" OR "motivational interviewing")] se modificó ligeramente en función de la configuración de cada base de datos.

\section{Criterios de inclusión}

Los estudios se seleccionaron considerando criterios relativos a los programas y a los diseños de investigación utilizados. En concreto se incluyeron aquellos programas que:

- incorporan la EM en cualquiera de sus modalidades.

- dirigidos a población adolescente y/o preadolescente, con edades entre 10 y 18 años.

- basados en la familia o con un componente familiar. Esto implica que se reconoce, desde su diseño, la importancia de la familia y se le asigna un papel protagonista durante la implementación del programa, ya sea como receptora directa de la intervención o participando parcialmente durante el programa.

- contienen un componente educativo o socioeducativo. Esto quiere decir que la parte educativa está presente, entendiendo la educación como aquella actividad que pretende desarrollar capacidades y habilidades en los participantes y que incluyen uno o varios de sus pilares: aprender a conocer, aprender a hacer, aprender a ser y aprender a estar- (Delors, 1996).

En cuanto a las características de los estudios y su diseño, se incluyeron estudios: publicados entre el 1999 y 2019.

- siguen una metodología experimental o cuasi-experimental con grupos de control o de comparación.

- $\operatorname{con} \mathrm{n}>1$.

- ofrecen resultados completos sobre la implementación, ya sean de eficacia, retención o adherencia.

Algunas de las razones de exclusión fueron: estudios de grupo único, diseños cualitativos y revisiones sistemáticas. También se excluyeron los estudios teóricos, sin resultados empíricos, con resultados preliminares o aquellos que no ofrecían resultados relacionados con la efectividad del programa ya sea en participación, adherencia o en relación al problema objetivo. Por ejemplo, quedaron excluidos aquellos estudios centrados en analizar el grado de ambivalencia de los asistentes en el programa, el grado de fidelidad de los profesionales en la implementación de la EM durante las sesiones y la formación de los formadores, entre otros. 


\section{Procedimiento de búsqueda}

En la Figura 1 se especifica el procedimiento de búsqueda y los resultados de las distintas fases en la selección de los estudios.

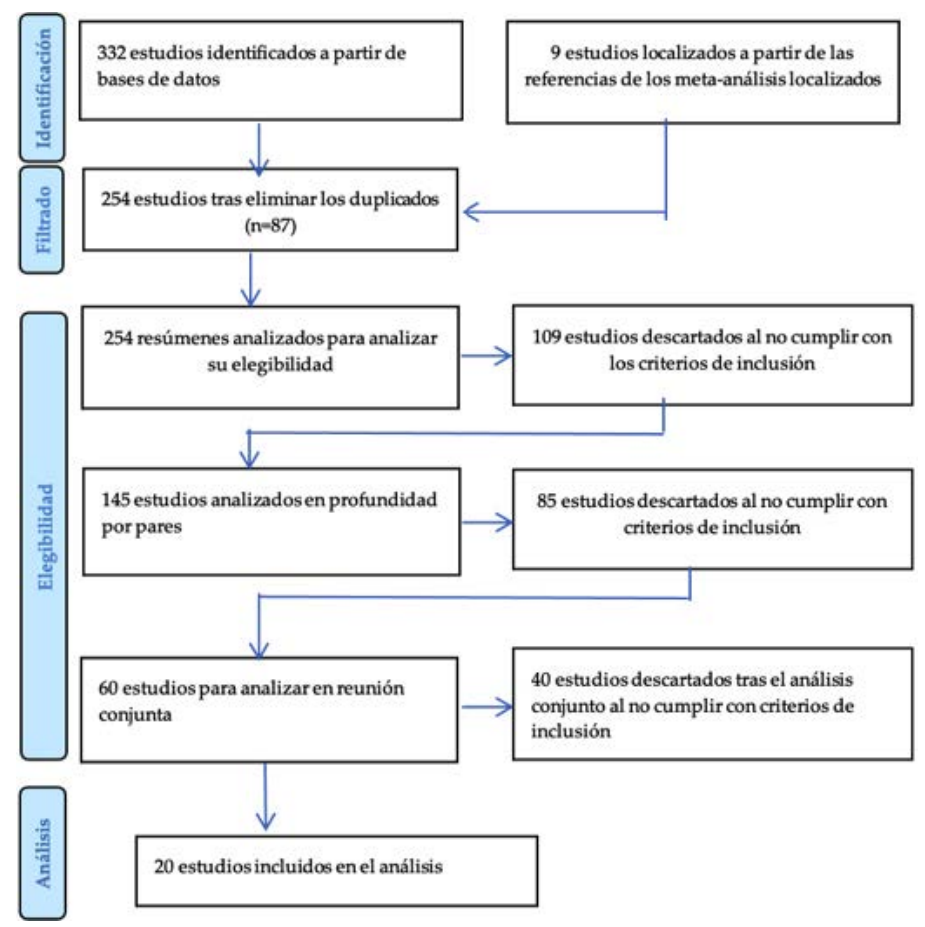

Figura 1. Procedimiento de búsqueda y selección de los artículos. Fuente: Elaboración propia, basado en la estructura ofrecida en el modelo PRISMA (Moher et al., 2009).

En la primera fase se encontraron 341 artículos, 332 provenientes de bases de datos y 9 de la revisión de las referencias disponibles en los meta-análisis localizados. Inicialmente se descartaron 87 artículos, al aparecer en diversas bases de datos y otros 109 al no cumplir con alguno de los criterios de inclusión. Los principales motivos de exclusión fueron la ausencia de un componente familiar o socioeducativo, así como la edad de la población objetivo.

Los 145 artículos restantes fueron leídos en mayor profundidad. Como resultado, se descartaron 85 estudios. Se analizaron en una reunión conjunta los 60 artículos restantes, culminando en 20 estudios seleccionados para el análisis.

\section{Fidelidad de la codificación}

Para asegurar la fidelidad en la codificación todos los artículos fueron revisados por pares. Se evaluó la idoneidad de cada artículo en función de los ocho criterios arriba mencionados. Si no se cumplía alguno de los criterios el artículo se rechazaba. Se uti- 
lizó el índice de Kappa de Fleiss' (Fleiss, 1971) para determinar el grado de acuerdo entre los juicos de las tres investigadoras en los 145 artículos. Se obtuvo un buen grado de acuerdo, k=.75 95\% CI[.519, .981], $p<.00$, si bien el grado óptimo se sitúa a partir de .80. Así, se revisaron conjuntamente en una reunión todos los artículos en los que las investigadoras marcaron provisionalmente aceptado o no coincidieran al marcar aceptado o rechazado.

\section{Resultados}

\section{¿Qué características tienen los programas y cuál es el formato de aplicación de la EM?}

En la Tabla 1 se muestran los 20 estudios, que hacen referencia a ocho intervenciones o programas socioeducativos con un componente familiar que usan la EM en alguna de sus modalidades.

Tabla 1

Estudios seleccionados, los programas analizados y sus características

\begin{tabular}{|c|c|c|c|c|c|c|c|c|}
\hline No & $\begin{array}{c}\text { Estudios } \\
\text { (autores, año) }\end{array}$ & $\begin{array}{c}N^{\circ} \text { de } \\
\text { estudios }\end{array}$ & $\begin{array}{c}\begin{array}{c}\text { Acrónimo } \\
\text { del } \\
\text { programa }\end{array} \\
\end{array}$ & $\begin{array}{l}\text { Programa o } \\
\text { intervención }\end{array}$ & $\begin{array}{c}\text { Tipo de } \\
\text { prevención }\end{array}$ & Temática & \begin{tabular}{c|}
$\begin{array}{c}\text { Contexto } \\
\text { de } \\
\text { aplicación }\end{array}$ \\
\end{tabular} & $\begin{array}{c}\text { Intervalo } \\
\text { de Edad }\end{array}$ \\
\hline 1 & $\begin{array}{c}\text { Dishion et al., } 2002 \\
\text { Connell et at., } 2007 \\
\text { Connell y Dishion, } \\
2008 \\
\text { Connell et al., } 2016 \\
\text { Stormshak et al., } 2009 \\
\text { Stormshak et al., } 2011 \\
\text { Van Ryzin y Dishion, } \\
2012 \\
\text { Fosco et al., 2013 } \\
\text { Van Ryzin y Nowicka, } \\
2013 \\
\text { Caruthers et al., 2014 } \\
\text { Fosco et al., 2015 } \\
\text { Connell et al., 2016 } \\
\text { Véronneau et al., } 2016\end{array}$ & 13 & ATP-FCU & $\begin{array}{l}\text { Adolescents } \\
\text { Transition Pro- } \\
\text { gram - Family } \\
\text { Check -up }\end{array}$ & $\begin{array}{l}\text { Universal/ } \\
\text { selectiva }\end{array}$ & $\begin{array}{l}\text { Conductas } \\
\text { problema } \\
\text { Consumo } \\
\text { de sustan- } \\
\text { cias }\end{array}$ & C.Escolar & $11-14$ \\
\hline 2 & MacDonell et al., 2012 & 1 & $\begin{array}{l}\text { Adap- } \\
\text { tación del } \\
\text { Healthy } \\
\text { choices }\end{array}$ & $\begin{array}{c}\text { Healthy } \\
\text { choices- } \\
\text { Adaptación } \\
\text { para prevenir } \\
\text { la obesidad con } \\
\text { componente } \\
\text { familiar }\end{array}$ & Selectiva & Obesidad & C.Salud & - \\
\hline 3 & Ball et al., 2011 & 1 & HIP & $\begin{array}{l}\text { Healthy initia- } \\
\text { tives program }\end{array}$ & $\begin{array}{l}\text { Selectiva/ } \\
\text { Indicada }\end{array}$ & Obesidad & C.Salud & $13-17$ \\
\hline 4 & Mayer-Davis et al. 2018 & 1 & FLEX & $\begin{array}{c}\text { The flex- } \\
\text { ible lifestyles } \\
\text { Empowering } \\
\text { change }\end{array}$ & $\begin{array}{l}\text { Selectiva/ } \\
\text { Indicada }\end{array}$ & Diabetes & C.Salud & $13-16$ \\
\hline 5 & Reinscow et al. 2005 & 1 & Go girls & Go girls & Selectiva & Obesidad & $\begin{array}{l}\text { Comuni- } \\
\text { dad } \\
\text { (iglesias) }\end{array}$ & $12-16$ \\
\hline
\end{tabular}




\begin{tabular}{|c|c|c|c|c|c|c|c|c|}
\hline $\mathrm{N}^{\mathrm{o}}$ & $\begin{array}{c}\text { Estudios } \\
\text { (autores, año) }\end{array}$ & $\begin{array}{c}N^{0} \text { de } \\
\text { estudios }\end{array}$ & $\begin{array}{c}\begin{array}{c}\text { Acrónimo } \\
\text { del } \\
\text { programa }\end{array} \\
\end{array}$ & $\begin{array}{l}\text { Programa o } \\
\text { intervención }\end{array}$ & $\begin{array}{c}\text { Tipo de } \\
\text { prevención }\end{array}$ & Temática & $\begin{array}{c}\text { Contexto } \\
\text { de } \\
\text { aplicación }\end{array}$ & $\begin{array}{c}\text { Intervalo } \\
\text { de Edad }\end{array}$ \\
\hline 6 & Naar et al. 2019 & 1 & $\begin{array}{l}\text { Interven- } \\
\text { ción } 6\end{array}$ & $\begin{array}{l}\text { Intervención } \\
\text { para reducción } \\
\text { de peso en } \\
\text { adolescentes } \\
\text { afroamericanas }\end{array}$ & Indicada & Obesidad & $\begin{array}{l}\text { C.Salud/ } \\
\text { hogar }\end{array}$ & $12-17$ \\
\hline 7 & Chahal et al., 2017 & 1 & $\begin{array}{l}\text { Interven- } \\
\text { ción } 7\end{array}$ & $\begin{array}{l}\text { Intervención } \\
\text { para cambiar el } \\
\text { estilo de vida } \\
\text { de adolescen- } \\
\text { tes dislipli- } \\
\text { démicos }\end{array}$ & Indicada & Obesidad & C.Salud & $10-17$ \\
\hline 8 & Nansel et al., 2015 & 1 & $\begin{array}{l}\text { Interven- } \\
\text { ción } 8\end{array}$ & $\begin{array}{l}\text { Intervención } \\
\text { para la mejora } \\
\text { de la dieta en } \\
\text { adolescentes } \\
\text { con diabetes } \\
\text { tipo I }\end{array}$ & Indicada & Diabetes & C.Salud & $8-17$ \\
\hline
\end{tabular}

De los 20 estudios, 13 hacen referencia al Adolescent Transition Program y al Family check-up (ATP-FCU) (Dishion et al., 2002; Connell et at. 2007; Connell \& Dishion, 2008; Stormshak et al., 2009; Stormshak et al., 2011; Van Ryzin \& Dishion, 2012; Fosco et al., 2013; Van Ryzin \& Nowicka, 2013; Caruthers et al., 2014; Fosco et al., 2015; Connell et al., 2016; Véronneau et al. 2016). Como se puede observar en la Tabla 1, el ATP-FCU es el único programa socioeducativo con componente familiar que usa la EM en prevención universal o primaria, el resto de programas son de prevención selectiva o indicada, ya que están dirigidos a grupos de riesgo, como Healthy Choices y Go girls (McDonell et al., 2012 y Reinscow et al., 2005), o a personas que ya presentan la problemática (Ball, 2001; Mayer-Davis, 2018; Naar et al., 2019; Chahal et al., 2017; Nansel et al., 2015).

En cuanto a la temática, destacan los estudios para la prevención de la obesidad, seguido de la diabetes y de la prevención de conductas problema y del consumo de sustancias (Tabla 1). Respecto al contexto de aplicación, el más frecuente es el centro de salud (Healthy Chioces; HIP en Ball, 2001;FLEX en Mayer-Davis, 2018; Intervención 6 en Naar et al., 2019; Intervención 7 en Chahal et al., 2017; e Intervención 8 en Nansel et al., 2015), realizándose también programas en el centro escolar (ATP-FCU), en la comunidad (Go girls), o en el propio hogar de los participantes (Intervención 6).

Los programas analizados presentan gran variabilidad en cuanto a duración y número de sesiones (Tabla 2). El número de sesiones fluctúa entre 3 y 30, desarrolladas entre 2 y 15 meses. Aplicándose la EM entre 3 (FCU) y 16 sesiones (HIP, FLEX). La EM se integra en todas las sesiones de los programas, exceptuando tres: Go girls, en el que se aplica en un máximo de 6 sesiones de las 30 de las que consta el programa, la Intervención 6 en la que se emplea en 10 de las 26 sesiones, y la intervención 7, desarrollándose en 4 sesiones.

Si bien la EM se aplica de forma integrada en la mayoría de los programas, encontramos variaciones en cuanto a su concepción, formato e incorporación (Tabla 2). En algunas intervenciones la EM tiene un papel preponderante, mientras en otros se integra como un componente añadido a un programa multicomponente previamente 
constituido. Este es el caso por ejemplo del programa Go Girls, en el que se combinan sesiones de EM con sesiones con otros objetivos. Por el contrario, en la intervención 6 y HIP la EM se considera un estilo de comunicación presente, o no, en función de la necesidad.

La duración de las sesiones de EM se sitúa entre 20 y 60 minutos. Respecto a la modalidad de aplicación, en todas se desarrolla de forma presencial, a excepción del programa Go girls que la aplica vía llamadas telefónicas.

Aunque la EM originalmente se desarrolló para aplicarla en adultos y de forma individual, en cuatro de las intervenciones analizadas se llevó a cabo en familia o de manera conjunta con uno de los padres o cuidadores (véase Tabla 2, FCU, Intervención 6 , Intervención 8); mientras que en tres se usó la EM solo con adolescentes (HIP, FLEX, Go Girls). En una de las intervenciones (Intervención 7), se aplicó a un grupo solamente con adolescentes y a otro conjuntamente con los padres, con el fin de analizar las diferencias de llevar a cabo la EM de forma individual con la persona adolescente o en familia.

Señalar también que únicamente tres de las intervenciones analizadas (Healthy Choices, $H I P$ y $F C U$ ) reportan el uso de un manual estructurado para la implementación en el seno del programa (Tabla 2). 


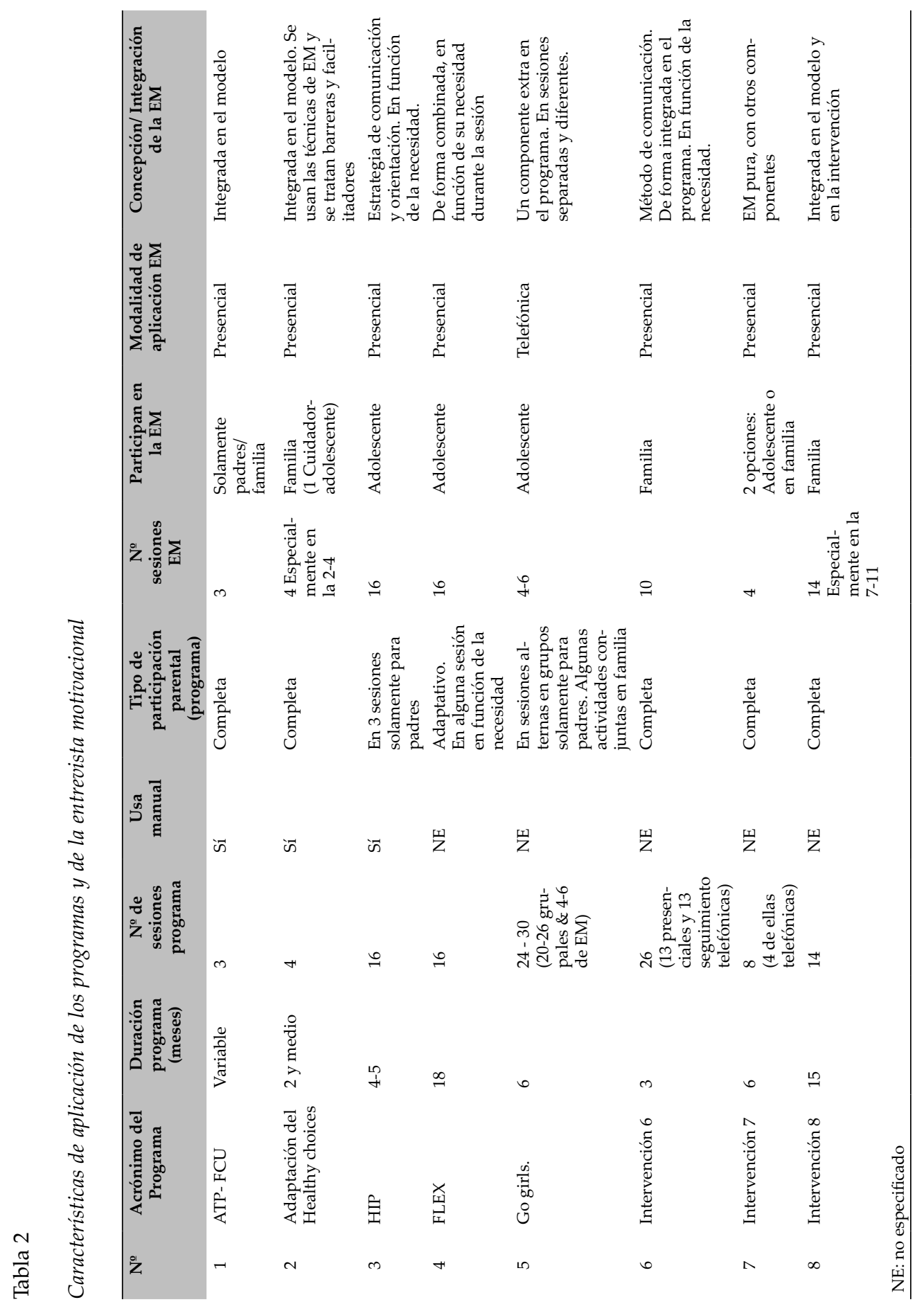




\section{¿Qué perfil y formación tienen los profesionales que aplican la entrevista motiva- cional en los programas?}

El perfil de los profesionales que implementan la EM es diverso (Tabla 3), siendo profesionales de la salud (médicos, asistentes, dietistas, terapeutas, psicólogos) y trabajadores comunitarios. En relación a la formación, las ocho intervenciones reportan realizar formación específica para los profesionales (Tabla 3).

Tabla 3

Perfil profesional y formación en EM

\begin{tabular}{|c|c|c|c|c|c|}
\hline $\mathrm{N}^{\mathrm{o}}$ & $\begin{array}{l}\text { Acrónimo del } \\
\text { Programa }\end{array}$ & $\begin{array}{l}\text { Perfil del profesional for- } \\
\text { mado }\end{array}$ & $\begin{array}{l}\text { Formación } \\
\text { en EM }\end{array}$ & $\begin{array}{c}\text { Duración de la } \\
\text { formación en EM }\end{array}$ & $\begin{array}{l}\text { Supervisión en } \\
\text { EM durante el } \\
\text { programa }\end{array}$ \\
\hline 1 & ATP- FCU & $\begin{array}{l}\text { Terapeuta } \\
\text { Profesor de universidad }\end{array}$ & Sí & NE & Sí \\
\hline 2 & $\begin{array}{l}\text { Adaptación del } \\
\text { Healthy choices }\end{array}$ & Dietista & Sí & 16 horas & Sí \\
\hline 3 & HIP & Profesional de la salud & Sí & 2 días & $\mathrm{NE}$ \\
\hline 4 & FLEX & Profesional de la salud & Sí & $\mathrm{NE}$ & Sí \\
\hline 5 & Go girls. & $\begin{array}{l}\text { Un dietista, un psicólogo y } \\
\text { counsellors }\end{array}$ & Sí & 16 horas & Sí \\
\hline 6 & Intervención 6 & $\begin{array}{l}\text { Trabajador comunitario } \\
\text { (para-profesional) y dietista }\end{array}$ & Sí & 300 horas & Sí (58 horas) \\
\hline 7 & Intervención 7 & $\mathrm{NE}$ & Sí & 6 días & Sí \\
\hline 8 & Intervención 8 & $\begin{array}{l}\text { Asistentes, becarios de in- } \\
\text { vestigación con formación } \\
\text { en diabetes tipo I pediátrica } \\
\text { y EM }\end{array}$ & Sí & $\mathrm{NE}$ & $\mathrm{NE}$ \\
\hline
\end{tabular}

$\mathrm{NE}=$ No especifica

En las cuatro intervenciones que indican la duración de la formación de los profesionales (Healthy Chocies, HIP, Intervención 6 y 7), ésta varía entre las 16 y las 300 horas. Además, como parte de la formación, seis intervenciones reportan realizar un seguimiento, evaluación y feedback sobre cómo los profesionales desarrollan la EM durante el programa, aspecto éste considerado clave en la literatura.

\section{¿Qué grado de eficacia tienen los programas analizados?}

En la Tabla 4 se muestran los datos referentes a los resultados de los programas. Solo uno de los estudios (programa HIP) ofrece el análisis de la eficacia diferencial, desagregando el efecto de la EM. Éste, a partir de dos grupos experimentales con intervenciones idénticas, con y sin EM, y grupo control, localiza diferencias estadísticamente significativas a favor de los grupos tratamiento (Ball et al., 2011). Si bien, 
los resultados no varían de forma estadísticamente significativa en función de que se aplique o no el componente de EM (Ibídem).

FCU, Adaptación del Healthy choices, FLEX e Intervención 8 presentan resultados positivos o moderadamente positivos al analizar los resultados del programa comparando con un grupo control. Uno de los programas, analiza las diferencias en función del grado de intensidad (Go girls, Reinscow et al., 2005), no observándose diferencias estadísticamente significativas entre los grupos. Por último, en la intervención 7 se analiza la diferencia entre el uso de EM solamente con adolescentes o en familia, distinguiendo una mejora en ambos grupos, aunque se obtienen ligeramente mejores resultados en la aplicación de la EM con adolescentes en solitario (Chahal et al., 2017).

Tabla 4

Grupos de comparación y resultados obtenidos en los programas

\begin{tabular}{|c|c|c|c|c|}
\hline № & $\begin{array}{c}\text { Acrónimo del } \\
\text { Programa }\end{array}$ & $\mathbf{n}$ & Grupos - comparación & Resultados \\
\hline 1 & ATP- FCU & $\begin{array}{l}998 \\
593\end{array}$ & $\begin{array}{l}2 \text { grupos } \\
- \text { GE } \\
- \text { GC }\end{array}$ & $\begin{array}{l}\text { Resultados positivos y con diferencias estadísti- } \\
\text { camente significativas en riesgo de obesidad, } \\
\text { suicidio, depresión, consumo de drogas y con- } \\
\text { ductas problema. }\end{array}$ \\
\hline 2 & $\begin{array}{l}\text { Adaptación } \\
\text { del Healthy } \\
\text { choices }\end{array}$ & 44 & $\begin{array}{l}2 \text { grupos: } \\
\text { - GE } \\
\text { - GC (Tr. habitual) }\end{array}$ & $\begin{array}{l}\text { Disminución estadísticamente significativa en el } \\
\text { consumo de comida rápida en el GE. } \\
\text { No hay cambios estadísticamente significativos } \\
\text { entre los grupos en el resto de variables. Tamaño } \\
\text { del efecto medio en la motivación intrínseca } \\
\text { para el ejercicio y en el consumo de verduras } \\
(\mathrm{d}>0,46)\end{array}$ \\
\hline 3 & HIP & 46 & $\begin{array}{l}3 \text { grupos: } \\
\text { - GE (HIP) } \\
\text { - GE (YLP) -semejante } \\
\text { al HIP sin EM- } \\
\text { - GC (lista de espera) }\end{array}$ & $\begin{array}{l}\text { Los resultados de la intervención HIP no difi- } \\
\text { eren estadísticamente de la YLP. Ambas inter- } \\
\text { venciones mejoran en relación al GC, aunque } \\
\text { modestamente. }\end{array}$ \\
\hline 4 & FLEX & 258 & $\begin{array}{l}2 \text { grupos: } \\
\text { - GE. } \\
\text { - GC (tr. habitual) }\end{array}$ & $\begin{array}{l}\text { La intervención no cambió de forma estadísti- } \\
\text { camente significativa los niveles de HbA de los } \\
\text { adolescentes, pero sí afectó positivamente a } \\
\text { niveles psicosociales de los participantes: Me- } \\
\text { jora de la motivación, Resolución de problemas, } \\
\text { Auto-gestión del perfil de diabetes, Informe } \\
\text { sobre la calidad de vida de los jóvenes, Miedo a } \\
\text { la hipoglucemia, conflicto familiar en diabetes } \\
\text { colesterol y presión sanguínea. }\end{array}$ \\
\hline 5 & Go girls. & 147 & $\begin{array}{l}2 \text { grupos: } \\
\text { - GE (EM de alta inten- } \\
\text { sidad) } \\
\text { - GE (de intensidad } \\
\text { moderada) }\end{array}$ & $\begin{array}{l}\text { No hay diferencias estadísticamente significati- } \\
\text { vas entre el grupo de alta intensidad con EM y } \\
\text { el de moderada intensidad, sin EM. Aunque se } \\
\text { observa una mejoría en el primero. } \\
\text { Aparecen diferencias estadísticamente signifi- } \\
\text { cativas entre los participantes del grupo de alta } \\
\text { intensidad que asisten al menos a un } 75 \% \text { de las } \\
\text { sesiones y el resto de participantes. }\end{array}$ \\
\hline
\end{tabular}




\begin{tabular}{|c|c|c|c|c|}
\hline № & $\begin{array}{c}\text { Acrónimo del } \\
\text { Programa }\end{array}$ & $\mathbf{n}$ & Grupos - comparación & Resultados \\
\hline 6 & Intervención 6 & 181 & $\begin{array}{l}\text { Varias opciones de trayec- } \\
\text { torias de intervención } \\
\text { posibles. }\end{array}$ & $\begin{array}{l}\text { En las diversas intervenciones disminuye el } \\
\text { sobrepeso en un } 3,2 \% \text {. }\end{array}$ \\
\hline 7 & Intervención 7 & 32 & $\begin{array}{l}2 \text { grupos: } \\
\text { - GE (EM para adoles- } \\
\text { cente) } \\
\text { - GE (EM para adoles- } \\
\text { cente + cuidador) }\end{array}$ & $\begin{array}{l}\text { Mejora en ambos GE, en comida saludable y } \\
\text { medidas clínicas. El grupo de adolescentes en } \\
\text { solitario obtuvo mejores resultados. }\end{array}$ \\
\hline 8 & Intervención 8 & 136 & $\begin{array}{l}2 \text { grupos: } \\
\text { - GE } \\
\text { - GC }\end{array}$ & $\begin{array}{l}\text { Mejora considerable en el GE en consumo de } \\
\text { comida saludable, calidad de la dieta. }\end{array}$ \\
\hline
\end{tabular}

Así, se observan mejoras en comportamientos saludables en los grupos experimentales respecto a los grupos de comparación, no obstante, no es posible desagregar si estos efectos se derivan de la inclusión de la EM en la intervención debido a la variedad de grupos de comparación.

\section{¿Qué grado de retención tienen los programas analizados?}

En líneas generales puede apreciarse que la asistencia y retención son relativamente elevadas (Tabla 5).

Tabla 5

Grado de retención y/o asistencia de los participantes en el programa

\begin{tabular}{|c|c|c|c|c|c|}
\hline № & $\begin{array}{c}\text { Acrónimo del } \\
\text { Programa }\end{array}$ & $\mathbf{N}$ & Medida & Resultados & $\begin{array}{c}\text { Diferencias estadísti- } \\
\text { camente significativas } \\
\text { entre grupos }\end{array}$ \\
\hline \multirow[t]{4}{*}{1} & ATP- FCU & $\begin{array}{l}\text { Muestra 1: } \\
500(\mathrm{GE})\end{array}$ & Captación en FCU & $\begin{array}{l}\text { Muestra 1. } \\
224(44,8 \%)\end{array}$ & NA \\
\hline & & $\begin{array}{l}\text { Muestra 2: } \\
386(\mathrm{GE})\end{array}$ & $\begin{array}{l}\text { № de familias que } \\
\text { consulta FRC }\end{array}$ & $\begin{array}{l}\text { Muestra 2: } \\
197(52,44 \%)\end{array}$ & \\
\hline & & & $\begin{array}{l}\text { № que participan en } \\
\text { FCU }\end{array}$ & $163(42,23 \%)$ & \\
\hline & & & $\begin{array}{l}\text { № que continua en } \\
\text { indicada }\end{array}$ & $131(34,20 \%)$ & \\
\hline \multirow[t]{2}{*}{2} & $\begin{array}{l}\text { Adaptación } \\
\text { del Healthy }\end{array}$ & $\begin{array}{l}22(\mathrm{GE}) \\
22(\mathrm{GC})\end{array}$ & $\begin{array}{l}\text { Asistencia a todas las } \\
\text { sesiones }\end{array}$ & $\begin{array}{l}\text { GE: } 27 \% \\
\text { GC: } 36,4 \%\end{array}$ & No \\
\hline & & & $\begin{array}{l}\text { Asistencia a } 2 \text { o más } \\
\text { sesiones }\end{array}$ & $\begin{array}{l}\text { GE: } 68 \% \\
\text { GC: } 81,8 \%\end{array}$ & No \\
\hline 3 & HIP & $\begin{array}{l}17(\mathrm{GE}) \\
10(\mathrm{GE}) \\
14(\mathrm{GC})\end{array}$ & Retención & $\begin{array}{l}\text { GE1: } 58,82 \% \\
\text { GE2: } 60 \% \\
\text { GC: } 78,57 \%\end{array}$ & No \\
\hline
\end{tabular}




\begin{tabular}{|c|c|c|c|c|c|}
\hline № & $\begin{array}{l}\text { Acrónimo del } \\
\text { Programa }\end{array}$ & $\mathbf{N}$ & Medida & Resultados & $\begin{array}{l}\text { Diferencias estadísti- } \\
\text { camente significativas } \\
\text { entre grupos }\end{array}$ \\
\hline 4 & FLEX & $\begin{array}{l}130 \text { (GE) } \\
128(\mathrm{GC})\end{array}$ & Retención & $\begin{array}{l}\text { GE: } 90,77 \% \\
\text { GC: } 100 \%\end{array}$ & $\mathrm{NE}$ \\
\hline 5 & Go girls. & 147 & $\begin{array}{l}\text { Retención } \\
\text { Asistencia }\end{array}$ & $\begin{array}{l}\text { Ambos grupos: } \\
84 \% \text {. } \\
\text { 57\% de las sesio- } \\
\text { nes en el GE1 (Alta } \\
\text { intensidad \& EM) } \\
66 \% \text { de las ses- } \\
\text { iones GE2 (Baja } \\
\text { intensidad, sin } \\
\text { EM) } \\
66,6 \%\end{array}$ & NE \\
\hline 6 & Intervención 6 & 181 & NA & NA & NA \\
\hline 7 & Intervención 7 & $\begin{array}{l}16 \text { (GE) } \\
16(\mathrm{GC})\end{array}$ & Retención & $\begin{array}{l}\text { GE: } 100 \% \\
\text { GC: } 100 \%\end{array}$ & No \\
\hline 8 & Intervención 8 & $\begin{array}{l}66 \text { (GE) } \\
70(\mathrm{GC})\end{array}$ & Retención & $\begin{array}{l}\text { GE: } 83,4 \% \\
\text { GC: } 100 \%\end{array}$ & NE \\
\hline
\end{tabular}

Dos de las intervenciones, Intervención 7 y FLEX tienen una retención muy elevada, superior al $90 \%$. Seguidos de Go girls y la Intervención 8, con una retención superior al $80 \%$. En el otro extremo encontramos el caso de HIP; este estudio muestra que no existen diferencias estadísticamente significativas en la retención entre los tres grupos -intervención con EM, intervención sin EM y control-, obteniendo una pérdida similar las intervenciones con EM y sin EM, si bien deben considerarse estos resultados con cautela debido al limitado tamaño de las muestras (Tabla 5).

Referente a la asistencia, el estudio de MacDonell y colaboradores (2012) (Adaptación del Healthy Choices), con una muestra muy pequeña, presenta una asistencia ligeramente menor en el grupo experimental que en el control, aunque no existen diferencias estadísticamente significativas entre ambos grupos, siendo la asistencia relativamente baja en ambos (Tabla 5). Asimismo, Reinscow y colaboradores (2005) (Go Girls) no reportan diferencias estadísticamente significativas entre los grupos, mostrando una asistencia ligeramente inferior en el grupo de alta intensidad con EM que en el de moderada intensidad. Mas, al tener un número diferente de sesiones, no son comparables (Tabla 5).

Los estudios relativos a FCU e Intervención 6, no proporcionan información relativa a los niveles de retención ni asistencia, aunque FCU sí de captación, siendo elevada.

Indagando la asistencia y grados de retención ofrecidos en los estudios, no parecen existir diferencias entre los grupos. Se observa un menor grado de retención en los grupos experimentales que en los controles, aunque no es estadísiticamente significativa, esto puede no tener relación con los programas o la EM y deberse al simple hecho de que los experimentales participan en una intervención mientras que los controles no la reciben. Indicar que el tipo de estudios disponibles y su variabilidad no permiten analizar si la EM mejora el grado de asistencia y retención en las intervenciones analizadas. 


\section{Discusión y conclusiones}

Este estudio muestra qué programas existen en prevención familiar con adolescentes con un componente socioeducativo que integran la EM, además analiza detalladamente cómo son, explorando sus características, duración, número de sesiones de EM y la forma en que se integra; compara el perfil y la formación específica en EM de los profesionales que imparten los programas y contrasta sus resultados en retención y eficacia.

Se han localizado ocho programas, siete de prevención selectiva o indicada, para participantes en situación de riesgo o vulnerabilidad o que ya han desarrollado la problemática y uno de prevención primaria. La mayoría de los programas localizados provienen del ámbito de la salud. El ámbito escolar está poco representado, apenas una experiencia, lo que muestra que estos programas son todavía escasos. Aparece también el propio hogar de los participantes como espacio para la formación familiar, pudiendo suponer un nicho para futuros programas.

La EM se aplica de forma integrada en la mayoría de casos, a pesar de que existen divergencias en su concepción, formato e incorporación. En algunas intervenciones se concibe la EM como un elemento central y básico del diseño mientras en otros supone un componente añadido. Asimismo, destaca la aplicación de forma presencial, apareciendo en formato telefónico en uno de los programas, en el que combinan sesiones educativas presenciales con sesiones individuales de EM telefónicas para el seguimiento y revisión de objetivos.

Se observa gran variabilidad en cuanto a duración y número de sesiones de los programas, entre 3 y 30, así como de las sesiones en las que se aplica la EM. El número predominante de sesiones de EM es de cuatro, aunque oscilan entre las 3 y las 16 sesiones. Este número elevado se debe principalmente a la presencia de programas que utilizan la EM como un estilo de comunicación dentro del mismo programa (p.ej. HIP), y al hecho de que en algunos se indica que está presente en todas las sesiones, aunque posteriormente se aplica, o no, en función de las necesidades de la familia y/o adolescente. Por ejemplo, Ball y colaboradores (2011) indican la conveniencia de utilizar la EM solamente cuando los cambios en los participantes son bajos o cuando ocurren retrocesos durante el proceso.

En relación a quién o quiénes reciben las sesiones de EM, se localizan programas que usan la EM con adolescentes y cuidadores por separado o la familia en conjunto, es decir, tanto en formatos grupales como individuales. Considerar que, si bien fue concebida como estrategia individual, la EM ha demostrado también ser útil como estrategia grupal tanto en adultos como en adolescentes en diversos ámbitos (Wagner e Ignersol, 2013). Esto apoyaría la idea de que la EM se puede adaptar a diferentes modalidades en función de los objetivos de la intervención sin perder eficacia.

Existe variabilidad con respecto al perfil del profesional que implementa la EM en los programas, mostrando como indican Schumaher y colaboradores (2014) que puede aplicarse por parte de diferentes perfiles, no necesariamente psicólogos, también educadores y trabajadores sociales, siempre que se cuente con la formación adecuada. La formación y las competencias profesionales son un elemento clave para el éxito de las intervenciones socioeducativas (Pascual et al., 2019). De hecho, 
en todos los estudios, los profesionales han recibido formación específica en EM, teniendo la mayoría sesiones de seguimiento de dicha formación como forma de garantizar que se aplica la EM con cierta calidad durante los programas. Ciertamente, para desarrollar una buena habilidad en EM es necesario no solamente la adquisición de competencias, sino también de una práctica continuada y supervisada de estas (Madson et al., 2009; Quesada et al., 2021).

Acerca de los resultados en asistencia, retención y eficacia, no se puede proporcionar una respuesta concreta. Los datos sugieren que los programas preventivos socioeducativos familiares con adolescentes con EM obtienen resultados positivos en eficacia. En todos los casos existe una mejora estadísticamente significativa respecto a los grupos de comparación, mas, es preciso recordar que analizar la eficacia de los programas en relación a la conducta que se quiere prevenir desagregando los efectos derivados de la EM, en función de los resultados disponibles en los estudios resulta complejo. No únicamente por la diversidad de objetivos, sino por el diseño (solamente un estudio, $H I P$, comparara el mismo programa con y sin EM), el tamaño de las muestras de algunos estudios y la falta de grupo control en otros.

Respecto a la participación y retención no se han detectado diferencias estadísticamente significativas entre los grupos en los que se participaba en el programa y los de comparación. La literatura en otros ámbitos indica que la EM puede ayudar a la retención de los participantes, fomentando la adherencia a la intervención (Herman et al., 2011; Hettema et al., 2005; Miller y Rose, 2009). Este aspecto no se ha podido analizar debido a las limitaciones mencionadas.

Este estudio permite mostrar una imagen de cómo se está aplicando la EM en programas familiares con adolescentes, y abre nuevos interrogantes ¿la incorporación de la EM en intervenciones socioeducativas ya existentes mejora la eficiencia, la participación o retención en dichas intervenciones? Se ha observado que en algunos programas se aplica la EM en sesiones concretas, mientras que en otros se aplica en función de la necesidad ¿cuándo y en qué formato es más aconsejable que se aplique la EM en los programas socioeducativos familiares? En relación a la formación de los profesionales que la aplican, se observa que tiene un papel relevante, sin embargo, ¿qué formación -formato y duración- es necesaria para la implementación de la EM por parte de profesionales del ámbito educativo y socioeducativo?

Una vez más, la investigación educativa y la difusión de los resultados de las experiencias y aplicaciones singulares, a partir de informes transparentes sobre cómo se aplica la EM en las intervenciones, así como el proceso que se ha seguido resultará clave en el futuro para responder a estos interrogantes. Explorar las opciones que ofrece la EM en el ámbito educativo y en la prevención de conductas problema o poco saludables es prometedora. Se hace necesario el estudio en profundidad de las intervenciones prácticas de la EM en el ámbito educativo y socioeducativo, su diseño y su evaluación, con la finalidad última de analizar y comparar el efecto concreto que produce la EM en este ámbito, como ya viene introduciéndose en otros espacios, y contribuir de este modo a una educación basada en la evidencia (Davies, 1999). 


\section{Referencias}

Ball, G.D.C. , Mackenzie-Rife, K.A., Nwton, M.S., Alloway, C.A., Slack, J.M., Plotnikoff, R.C., y Goran, M.I. (2011). One-on-one lifestyle coaching for managing adolescent obesity: Findings from a pilot, randomized controlled trial in a real-world, clinical setting. Pediatric child health, 16, 345-350. https://doi.org/10.1093/pch/16.6.345

Ballester, L., Orte, C., y Negreiros, J. (2021). La entrevista motivacional y su aplicabilidad en los programas socioeducativos familiares. Tirant lo Blanch.

Barnett, E., Sussman, S., Smith, C., Rohrbach, L.A., y Spruijt-Metz, D. (2012). Motivational interviewing for adolescent substance use: A review of the literature. Addictive behaviors, 37(12), 1325-1334.

Belmontes, K.C. (2018). When Family Gets in the Way of Recovery. The Family Journal, 26(1), 99-104. doi:10.1177/1066480717753013

Caruthers, A.S., Van Ryzin, M.J., y Dishion, T.J. (2014). Preventing high-risk sexual behavior in early adulthood with family interventions in adolescence: outcomes and developmental processes. Prevention Science, suplemento 1, 59-69. https://doi. org/10.1007/s11121-013-0383-9

Connell, A.M., Dishion, T.J., Yasui, M., y Kavanagh, K. (2007). An adaptive approach to family intervention: Linking engagement in family-centered intervention to reductions in adolescent problem behavior. Journal of Consulting and Clinical Psychology, 75(4), 568-579. https://doi.org/10.1037/0022-006X.75.4.568

Connell, A.M., y Dishion, T.J. (2008). Reducing depression among at-risk early adolescents: Three-year effects of a family-centered intervention embedded within schools. Journal of Family Psychology, 22(4), 574-585. https://doi.org/10.1037/08933200.22.3.574

Connell, A.M., McKillop, H.N., Dishion, T.J., Van Ryzin, M.J., Connell, A.M., y Stormshak, E.A. (2016). Long-term effects of the family check-up in early adolescence on risk of suicide in early adulthood. Suicide Life Threat Behaviour, Suplemento 1, 15-22. https://doi.org/10.1111/sltb.12254

Chahal, N., Rush, J., Manlhiot, C., Boydell, K.M., Jelen, A., y McCrindle, B.W. (2017). Dyslipidemia management in overweight or obese adolescents: A mixed-methods clinical trial of motivational interviewing. SAGE Open Medicine, 5, 1-11. https://doi. org/10.1177/2050312117707152

Cushing, C.C., Jensen, C.D., Miller, M.B., y Leffingwell, T.R. (2014). Meta-analysis of motivational interviewing for adolescent health behavior: Efficacy beyond substance use. Journal of Consulting and Clinical Psychology, 82(6), 1212- https://doi. org/1218. 10.1037/a0036912

Davies, P. (1999). What Is Evidence-Based Education?. British Journal of Educational Studies, 47(2), 108-121.

Delors, J. (1996). Los cuatro pilares de la educación. En UNESCO (Eds). La educación encierra un tesoro. Informe a la UNESCO de la Comisión internacional sobre la educación para el siglo XXI (pp. 91-103). Santillana/UNESCO.

Dishion, T.J., Kavanagh, K., Schneiger, A., Nelson, S., y Kaufman, N.K. (2002). Preventing early adolescent substance use: A family-centered strategy for the public middle school. Prevention Science, 3(3), 191-201. https://doi.org/10.1023/a:1019994500301 
Fosco, G.M., Frank, J.L., Stormshak, E.A., y Dishion, T.J. (2013). Opening the "Black Box": family check-up intervention effects on self-regulation that prevents growth in problem behavior and substance use. Journal of School Psychology, 31(4), 455-468. https://doi.org/10.1016/j.jsp.2013.02.001

Fosco, G.M., Van Ryzin, M.J., Connell, A.M., y Stormshak, E.A. (2015). Preventing adolescent depression with the family check-up: Examining family conflict as a mechanism of change. Journal of Family Psychology, 30(1), 82-92. https://doi.org/10.1037/ fam0000147

Galevez Espinoza, P., Gómez San Carlos, N., Nicoletti Rojas, D., y Cerda Rioseco, R. (2019). ¿Es efectiva la entrevista motivacional individual en la malnutrición por exceso? Una revision sistemática de la literature. Atención Primaria, 51(9), 548-561.

Gayes, L.A., y Steele, R.G. (2014). A meta-analysis of motivational interviewing interventions for pediatric health behavior change. Journal of consulting and clinical psychology, 82(3), 521. https://doi.org/10.1037/a0035917

Gearing, R.E., Townsend, L., Elkins, J., El-Bassel, N., y Osterberg, L. (2014). Strategies to Predict, Measure, and Improve Psychosocial Treatment Adherence. Harvard Review of Psychiatry, 22(1), 31-45. https://doi.org/10.1097/hrp.10.1097/hrp.0000000000000005

Herman, K. C., Borden, L. A., Hsu, C., Schultz, T. R., Strawsine Carney, M., Brooks, C. M., y Reinke, W. M. (2011). Enhancing Family Engagement in Interventions for Mental Health Problems in Youth. Residential Treatment for Children \& Youth, 28(2), 102-119. https://doi.org/10.1080/0886571X.2011.569434

Hettema, J., Steele, J., y Miller, W.R. (2005). Motivational interviewing. Annual Review of Clinical Psychology, 1, 91-111.

Jensen, C.D., Cushing, C.C., Aylward, B.S., Craig, J.T., Sorell, D.M., y Steele, R.G. (2011). Effectiveness of motivational interviewing interventions for adolescent substance use behavior change: a meta-analytic review. Journal of consulting and clinical psychology, 79(4), 433. https://doi.org/10.1037/a0023992

Lundahl, B.W., Kunz, C., Brownell, C., Tollefson, D., y Burke, B.L. (2010). A metaanalysis of motivational interviewing: Twenty-five years of empirical studies. Research on Social Work Practice, 20(2), 137-160. https://doi.org/10.1177/1049731509347850.

Madson, B., M., Loignon, A.C., y Lane, C. (2009). Training in motivational interviewing: A systematic review. Journal of substance Abuse Treatment, 36, pp. 101-109.

MacDonell, K, Brogan, K., Naar-King, S., Ellis, D., y Marshall, S. (2012). A pilot study of Motivational interviewing targeting weight-related behaviors in Overweight or Obese African American Adolescents. Journal of Adolescent Health, 50, 201-203. https:// doi.org/10.1016/j.jadohealth.2011.04.018.

Mayer-Davis, E.J., Maahs, D., Seid, M., Crandell, J., Bishop, F.K., Driscoll, K.A., Hunter, C.M., Kichler, J.C., Standiford, D., Thomas, J.M., y FLEX study Group. (2018). Efficacy of the Flexible Lifestyles Empowering Change intervention on metabolic and psychosocial outcomes in adolescents with type 1 diabetes (FLEX): a randomised controlled trial. Lancet Child and Adolescent Health, 2(9), 635-646. https://doi. org/10.1016/S2352-4642(18)30208-6

Miller, W.R., y Rollnick, S. (2015) La entrevista motivacional: Ayudar a las personas a cambiar. Ediciones Paidós 
Miller, W. R., y Rose, G. S. (2009). Toward a theory of motivational interviewing. American Psychologist, 64(6), 527-537. https://doi.org/10.1037/a0016830

Moher D, Liberati A, Tetzlaff J, Altman DG, The PRISMA Group. (2009). Preferred Reporting Items for Systematic Reviews and Meta-Analyses: The PRISMA Statement. BMJ, 339 :b2535. https://doi.org/10.1136/bmj.b2535

Naar, S., Ellis, D., Idalski-Carcone, A., Jacques-Tiura, A.J., Cunningham, P., Templin, T., Brogan Hartlieb, KL., y Jen, K.-L.C. (2019). Outcomes from a sequential multiple assignment randomized trial of weight loss strategies for african american adolescents with obesity. Annals of Behavioral Medicine, 53(10), 928-938. https://doi. org/10.1093/abm/kaz003

Nansel, T.R., Laffel, L.M.B., Haynie, D.L., Mehta, S.N., Lipsky, L.M., Volkening, L.K., Butler, D.A., Higgins, L.A., y Liu, A. (2015). Improving dietary quality in youth with type 1 diabetes: randomized clinical trial of a family-based behavioral intervention. International Journal of Behavioral Nutrition and Physical Activity, 12, 1-11. https://doi.org/10.1186/s12966-015-0214-4

Negreiros, J., Ballester, L., Valero, M., Carmo, R., y da Gama, J. (2019). Una revisión sistemática de la participación en los programas de prevención familiar. Pedagogía Social. Revista Interuniversitaria, 34, 63-75. https://doi.org/10.7179/PSRI_2019.34.05

Norberg, M.M., Kezelman, S., y Lim-Howe, N. (2013). Primary prevention of cannabis use: A systematic review of randomized controlled trials. PLos ONE, 8(1). https:// doi.org/10.1371/journal.pone.0053187

Orte, C., Ballester, L., Vives, M., Amer, J., y March, M.X. (2017). Cultural adaptation of family evidence-based drug prevention programs in Spain. En M. Israelshivili y J. Romano (Eds.), Cambridge Handbook of International Prevention Science (pp. 873-895). Cambridge University Press.

Resnicow, K., Taylor, R., Baskin, M., y McCarty, F. (2005). Results of Go Girls: A weight control program for overweight African-American Adolescent Females. Obesity research, 13, 1739-1748. https://doi.org/10.1038/oby.2005.212

Pascual, B., Sánchez-Prieto, L., Gomila, M.A., Quesada, V. y Nevot, L. (2019). Formación para la prevención en el ámbito socioeducativo: un análisis de los perfiles profesionales. Pedagogía Social. Revista Interuniversitaria, 34, 31-44. https://doi.org/10.7179/ PSRI_2019.34.03

Quesada Serra, V., Pascual Barrio, B., Gomila Grau, M.A. (2021). La Formación en Entrevista Motivacional y su Conveniencia para el Desarrollo de Competencias en Profesionales de Programas Socioeducativos Familiars. En L. Ballester, C. Orte y J. Negreiros (pp.129-144). La entrevista motivacional y su aplicabilidad en los programas socioeducativos familiares. Tirant lo Blanch.

Schumaher, J.A., Madson, M.B., y Nilsen, P. (2014). Barriers to Learning Motivational Interviewing: A Survey of Motivational Interviewing Trainers' Perceptions. Journal of Addictions \& Offender Counseling, 45, 81-96. https://doi.org/10.1002/j.21611874.2014.00028.x

Shaw, D.S., y Taraban, L.E. (2016). New Directions and Challenges in Preventing Conduct Problems in Early Childhood. Child Development Perspectives, 11(2), 85-89. https:// doi.org/10.1111/cdep.12212 
Smith, J.D., Montaño, Z., Dishion, T.J., Shaw, D.S., Wilson, M.N. (2015). Preventing weight gain and obesity: indirect effects of the family check-up in early childhood. Prevention Science, 16(3), 408-419. https://doi.org/10.1007/s11121-014-0505-z.

Stormshak, E.A., Connell, A., y Dishion, T.J. (2009). An adaptive approach to familycentered intervention in schools: Linking intervention engagement to academic outcomes in middle and high school. Prevention Science, 10(3), 221-235. https://doi. org/10.1007/s11121-009-0131-3

Stormshak, E.A., Connell, A.M., Véronneau, M.H., Myers, M.W., Dishion, T.J., Kavanagh, K., y Caruthers, A.S. (2011). An ecological approach to promoting early adolescent mental health and social adaptacion: Family-centered intervention in public middle schools. Child Development, 82(1), 209-225. https://doi.org/10.1111/j.14678624.2010.01551.x

United Nations Office on Drugs and Crime (2015). Guide to implementing family skills training programmes for drug abuse prevention. United Nations publication.

United Nations Office on Drugs and Crime. (2018). International Standards on Drug Use Prevention. World Health Organisation Preventing youth violence: an overview of the evidence. United Nations publication.

Van Ryzin, M.J., Stormshak, E.A., y Dishion, T.J. (2012). Engaging parents in the family check-up in middle school: longitudinal effects on family conflict and problem behavior through the high school transition. Journal of Adolescents Health, 50(6), 627-633. https://doi.org/10.1016/j.jadohealth.2011.10.255

Van Ryzin, M.J., y Nowicka, P. (2013). Direct and indirect effects of a family-based intervention in early adolescence on parent-youth relationship quality, late adolescent health, and early adult obesity. Journal of Family Psychology, 27(1), 106-116. https://doi.org/10.1037/a0031428

Véronneau, M.H., Dishion, T.J., Connell, A.M., y Kavanagh, K. (2016). A randomized, controlled trial of the family check-up model in public secondary schools: Examining links between parent engagement and substance use progressions from early adolescence to adulthood. Journal of Consulting and Clinical Psychology, 84(6), 526-543. https://doi.org/10.1037/a0040248

Wagner, C.C., y Ingersoll, K.S. (2013). Motivational Interviewing in Groups. The Guilford Press.

Wells, H., Jones, A., Jones, C. (2014). Teaching reluctant students: using the principles and techniques of motivational interviewing to foster better student-teacher interactions. Innovations in Education and Teaching International, 51(1), 111-118. https:// doi.org/10.1080/14703297.2013.778066

Wells, H. y Jones, A. (2018). Learning to change: the rationale for the use of motivational interviewing in higher education. Innovations in Education and Teaching International, 55(1), 111-118. https://doi.org/10.1080/14703297.2016.1198714

Fecha de recepción: 1 de julio de 2020.

Fecha de revisión: 10 de septiembre de 2020.

Fecha de aceptación: 17 de marzo de 2021. 